\title{
Low-density optical pump - THz probe analysis of high-temperature superconductors
}

\author{
M. Brucherseifer, A. Meltzow-Altmeyer, P. Haring Bolivar, and H. Kurz \\ Institut für Halbleitertechnik, RWTH Aachen, Sommerfeldstr. 24, 52056 Aachen, Germany \\ E-mail: mbrucher@stevens-tech.edu
}

\begin{abstract}
Using time-resolved $\mathrm{THz}$ probing we analyze the dynamics of low-density optically excited, optimally doped $\mathrm{YBa}_{2} \mathrm{Cu}_{3} \mathrm{O}_{7-\delta}$. A previously unknown additional relaxation component is found, tentatively indicating the separate relaxation of charge and spin excitations.
\end{abstract}

\section{Introduction}

Dynamic analysis of the relaxation dynamics of high-temperature superconductors (HTSC) after optical excitation offer a detailed insight into the superconductivity mechanism of these materials. Spectrally similar effects which would be superposed in stationary experiments can readily be distinguished in the time domain. In the past, most time-resolved experiments on HTSC have probed high energy states at photon energies around 1.0- $2.0 \mathrm{eV}$ [1]. However, under these conditions, superconductivity related pairing dynamics cannot be distinguished from energy relaxation dynamics, as the probing energy is too far above the superconductivity gap. Recent experiments, probing pairing dynamics at lower energies e.g. MIR [2] or $\mathrm{THz}$ [3], have yielded interesting observations. However, these experiments were performed under very high optical excitation densities, which significantly modify the superconducting state [3]. Here we present a time-resolved optical pump-THz probe analysis with a drastically improved resolution better than $\Delta \mathrm{T} / \mathrm{T}_{0}=10^{-9}$, which allows to perform analysis at excitation densities 2 - 3 orders of magnitudes below previous approaches. Thus density dependent recombination dynamics in optimally doped $\mathrm{YBa}_{2} \mathrm{Cu}_{3} \mathrm{O}_{7-\delta}$ probed at low energies are isolated for the first time. In contrast to a previously seen single relaxation component, two components are observed, tentatively indicating the separate relaxation of spin and charge excitations.

\section{Experimental Methods}

The experiments are performed with a time-domain $\mathrm{THz}$ transmission setup using a $76 \mathrm{MHz}$ repetition rate $100 \mathrm{fs}$ laser. Its great advantage is the possibility to directly measure the complex conductivity $\sigma=\sigma_{1}-i \sigma_{2}$ of HTSC [4]. Based on a Two-Fluid-Model [5], one can simultaneously and separately analyze the behavior of quasiparticle $\mathrm{x}_{\mathrm{n}}$ and superconducting condensate $\mathrm{x}_{\mathrm{s}}$ densities. The samples used consist of optimally doped $20 \mathrm{~nm} \mathrm{YBa}_{2} \mathrm{Cu}_{3} \mathrm{O}_{7-\delta}$ films on $\mathrm{NdGaO}_{3}$ substrates mounted in a cryostat. A stationary, temperature dependent evaluation of the complex 
conductivity delivers a critical temperature of $\mathrm{T}_{\mathrm{C}}=88 \mathrm{~K}$ confirming a good sample quality despite its thin thickness. For dynamical analysis a further optical laser pulse is focused, collinear to the $\mathrm{THz}$ beam, onto the sample with a $800 \mu \mathrm{m}$ focus (= THz focus). Primary excitation densities in the range of $3 \cdot 10^{16}$ to $4.5 \cdot 10^{17} \mathrm{~cm}^{-3}$ are induced. The optical excitation breaks Cooper-pairs resulting in a decrease of $\mathrm{x}_{\mathrm{s}}$ and increase of $\mathrm{x}_{\mathrm{n}}$, accordingly an increase of $\sigma_{1}$ and decrease of $\sigma_{2}$. The following relaxation process is dynamically analyzed by probing the $\mathrm{THz}$ transmission. A detailed numerical calculation, considering the optically altered conductivity due to the changed fractions of $\mathrm{x}_{\mathrm{n}}$ and $\mathrm{x}_{\mathrm{s}}$, leads to expected THz transmission intensity modifications of maximally $\Delta \mathrm{T} / \mathrm{T}_{0} \approx 10^{-8}$ which have to be resolved, compared to a free-space $\mathrm{THz}$ transmission $\mathrm{T}_{0}$. To avoid long data acquisition measurements we do not analyze the full dependence of the $\mathrm{THz}$ transmission as function of optical and $\mathrm{THz}$ time delays but concentrate only on characteristic fixed delay points of the $\mathrm{THz}$ transient, and evaluate the dependence of the optical time delay. As indicated on the $\mathrm{THz}$ transmission transient in the inset of Fig. 1, we concentrate on the conductivity dynamics at the marked peak and zero crossing positions. Our numeric calculation reveals, that the signal increase measured at the peak of the THz-transient is dominated by the decrease of $\sigma_{2}$, whereas the signal at the marked zero crossing increases, depends equally on the $\sigma_{1}$ increase and the $\sigma_{2}$ decrease.

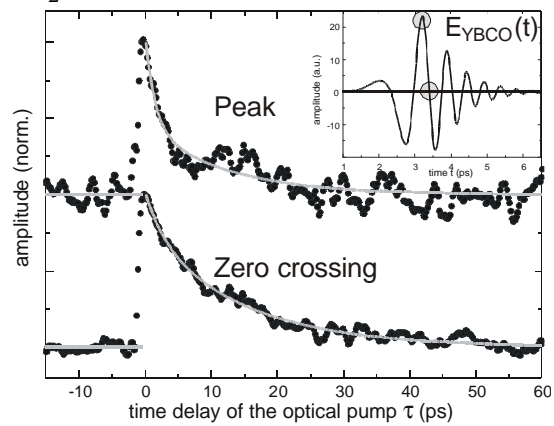

Fig 1. The inset shows a THz transient and the characteristic positions used for dynamical analysis. The main figure shows the respective relaxation signals after optical excitation.

\section{Results and Discussion}

Two exemplary relaxation transients taken at $10 \mathrm{~K}$ are shown in Fig. 1. One can directly see different relaxation times of both transients indicating that at least two different components are necessary to explain the relaxation process. As the zero crossing signal has a better signal to noise ratio and contains both dynamics, we restrict in the following to such analysis and evaluate these transients via two component exponential fits with decay times $\tau_{1}$ and $\tau_{2}$. Fig. 2 shows the analysis of the density and temperature dependencies of both components. The left plot indicates a fast component $\tau_{2}$ in the region of 1-3 ps that is in agreement with previous high-density observations [2,3]. This fast component exhibits a characteristic Rothwarf-Taylor density dependence. For the first time we observe an additional slow relaxation component $\tau_{1}$ which decreases for increasing excitation density. We think that this additional slow component was not previously observed, as pre- 

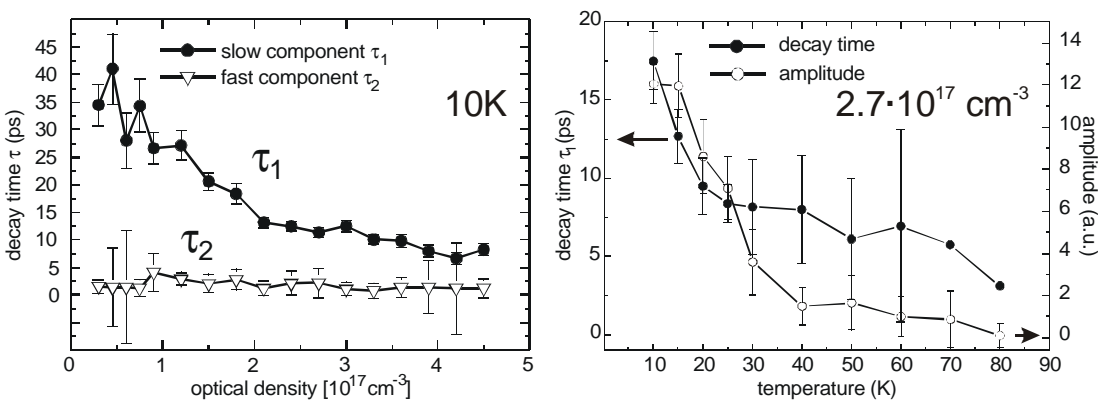

Fig2. Decay time density dependence of both components $\tau_{2}$ and $\tau_{2}$ at $10 \mathrm{~K}$ (left) and temperature dependence of amplitude and decay time of the slow component $\tau_{1}$ (right).

vious experiments were performed at much higher excitation densities where both relaxation channels $\tau_{1}$ and $\tau_{2}$ become restricted by phonon dissipation. The temperature dependence of the amplitude and relaxation time of $\tau_{1}$ are shown in the right part of Fig. 2. The decay time $\tau_{1}$ decreases for higher temperatures. While the amplitude of the fast component $\tau_{2}$ has a typical BCS type behavior with a sharp drop close to $\mathrm{T}_{\mathrm{C}}$ (data not shown), the $\tau_{1}$ component decays significantly already much below $\mathrm{T}_{\mathrm{C}}$.

\section{Conclusions}

The presence of two markedly different relaxation components proofs that the simple assumption of a single type of excitations is clearly not sufficient to explain the observed dynamics. We tentatively ascribe both components to the separate relaxation of charge and spin excitations (antiferromagnetic spin fluctuations), supporting the notion of independent charge and spin excitations within a collective state, the "quantum protectorate" [6]. The decrease of the respective relaxation times with increasing excitation density would then reflect the increasing probability for the coincidence of charge or spinon pairs, respectively. Further experimental and theoretical analysis will however be necessary to further support this conjecture and to assign the respective components.

\section{References}

1 S. G. Han, Z. V. Vardeny, K. S. Wong, O. G. Symko, Phys. Rev. Lett. 65, 2708 (1990).

2 R. A. Kaindl, M. Woerner, T. Elsaesser, D. C. Smith, J. F. Ryan, G. A. Farnan, M. P. McCurry and D. G. Walmsley. Science 287, 470 (2000).

3 R. D. Averitt, G. Rodriguez, A. I. Lobad, J. L. W. Siders, S. A. Trugman, and A. J. Taylor., Phys. Rev. Rapid Comm. 63, 140502 (2001).

4 S. D. Brorson, , R. Buhleier, I. E. Trofimov, J. O. White, C. Ludwig, F. F. Balakirev, H.-U. Habermeier, and J. Kuhl. J. Opt. Soc. Am. B 13, 1979 (1996).

5 A. J. Berlinsky, C. Kallin, G. Rose and A. C. Shi., Phys. Rev. B 48 (1993).

6 P. W. Anderson, Science 288, 480 (2000). 